\title{
Novel Adaptive Codebook-Based Limited Feedback Techniques for Multi-user MIMO-OFDM Systems
}

\author{
I-Tai Lu and Jiang Chang \\ Department of Electrical and Computer Engineering \\ Polytechnic Institute of New York University, Brooklyn, NY 11201, USA \\ itailu@rama.poly.edu, changjiang.cn@gmail.com
}

\begin{abstract}
To mitigate the sum rate ceiling problems, high-resolution quantization and feedback schemes for the channel state information (CSI) are highly demanded in multi-user (MU) multiple-input multiple-output (MIMO) orthogonal frequency division multiplexing (OFDM) systems. In this paper, we investigate three adaptive codebook-based limited feedback techniques for MU-MIMO-OFDM broadcast systems. Using the channel covariance and mean respectively, adaptive codebook techniques I and II are extensions of two adaptive codebook techniques proposed for single-user MIMO single-carrier systems. Technique III is a totally novel proposal wherein both channel covariance and mean are exploited in the adaptive codebook construction. Numerical results show that the adaptive codebook approaches have significant sum rate gain compared to the fixed codebook solution and technique III outperforms techniques I and II.
\end{abstract}

Index Terms - adaptive codebook, multiuser, MIMO, OFDM, limited feedback

\section{INTRODUCTION}

$\mathrm{M}$ UTIPLE-input multiple-output (MIMO) and orthogonal frequency division multiplexing (OFDM) have been adopted in many modern broadband wireless communication systems, such as IEEE 802.11n Wireless Local Area Networks (WLAN) [1], IEEE $802.16 \mathrm{~m}$ Wireless Metropolitan Area Networks (WiMAX) [2] and 3GPP Long Term Evolution (LTE) [3] systems. In MIMO communications, channel state information (CSI) needs to be known at the transmitter in order to achieve the maximal diversity and/or multiplexing gain. Accurate CSI is crucial since CSI error introduces the inter-data-stream interference and thus the sum rate ceiling problems. In frequency division duplex (FDD) systems, one widely used solution is to quantize the CSI at the receiver and send this information to the transmitter over a limited-rate feedback channel [4]. In a codebook-based quantization and limited feedback approach, the common codebook with size $N$ is available at both the transmitter and receiver. At the receiver, the CSI is quantized using the codebook and the index of the selected codeword is fed back to the transmitter with $\log _{2} N$ bits. Generally, a larger $N$ brings in a smaller CSI quantization error but a larger feedback overhead. Thus, the important thing is how to find a codebook with a reasonable $N$ but providing a small quantization error.

This work was supported in part by InterDigital.
Codebook design has been discussed extensively in many literatures (e.g., see [5-8]). Most of these codebooks are designed and optimized for uncorrelated Rayleigh fading channel without exploiting the channel spatial or temporal correlation. An adaptive codebook technique for the beamformer in single-user (SU) MIMO single-carrier systems is proposed in [9] to utilize the channel covariance of the correlated MIMO channel. Other adaptive codebook techniques for SU MIMO single-carrier systems are proposed in [10] and [11] where an adaptive local codebook for the beamformer is constructed through rotating and scaling a root codebook.

In this contribution, we investigate three adaptive codebook techniques for the multi-user (MU) MIMO-OFDM broadcast systems. Unlike the SU cases, in MU-MIMO broadcast systems, users do not have sufficient information to recommend the precoder assumed at the base station. Therefore, in MU cases, the adaptive codebooks are designed for channel (rather than beamformer) quantization and feedback. Adaptive codebook technique I and II are extensions of the SU-MIMO adaptive codebook techniques in [9]-[11]. Adaptive codebook technique III is a novel proposal wherein both the first- and second-order statistics of the channel are exploited in adaptive codebook construction to further improve the system performances. The sum rate performances of the three adaptive codebook techniques in MU-MIMO systems are investigated through simulations based on the LTE system profile. Compared to the fixed codebook solution, significant sum rate gains are achieved with adaptive codebook techniques. Furthermore, the novel proposed adaptive codebook technique (technique III) outperforms technique I and II in sum rate performance.

Throughout this paper, the transpose of a vector or matrix is denoted by $(\cdot)^{T}$, conjugate transpose by $(\cdot)^{H}$ and Matrix inversion is denoted by $(\cdot)^{-1} \cdot\|\cdot\|_{2}$ denotes the vector 2-norm. $(\cdot)^{\perp}$ denotes the null space of a vector. A scalar is denoted by a lower case character. A vector is denoted by a boldface lowercase character. A matrix is denoted by a boldface capital character.

\section{SYSTEM FORMULATION}

Consider a MU-MIMO-OFDM broadcast system. A base station equipped with $N_{t}$ transmit antennas will transmit to $K$ $\left(K \leq N_{t}\right)$ active users each with a single receive antenna. The 
receive signal on the $l$-th subcarrier of the $n$-th symbol for the $k$-th user is given by

$$
\begin{aligned}
& y_{k}(l, n)=\sqrt{\frac{P}{K}} \mathbf{h}_{k}^{T}(l, n) \mathbf{f}_{k}(l, n) s_{k}(l, n) \\
& +\sqrt{\frac{P}{K}} \mathbf{h}_{k}^{T}(l, n) \sum_{i \neq k}^{K} \mathbf{f}_{i}(l, n) s_{i}(l, n)+w_{k}(l, n)
\end{aligned}
$$

where $s_{k}$ is the transmit symbol, $y_{k}$ is the receive signal, $\mathbf{f}_{k}$ is the beamforming vector, $\mathbf{h}_{k}^{T}$ is the complex row vector of the MISO channel, $w_{k}$ is the complex Gaussian noise with distribution $N\left(0, \sigma^{2}\right)$, and $P$ is the total transmit power at the base station. For convenience, the subcarrier index $l$ and symbol index $n$ will be omitted when there will be no confusion.

Since the power is equally allocated to all $K$ users, the signal-to-interference-plus-noise ratio (SINR) at the $k$-th user is given by [12]

$$
\operatorname{SINR}_{k}=\frac{\frac{P}{K}\left|\mathbf{h}_{k}^{T} \mathbf{f}_{k}\right|^{2}}{\sigma^{2}+\frac{P}{K} \sum_{i \neq k}\left|\mathbf{h}_{k}^{T} \mathbf{f}_{i}\right|^{2}}
$$

and the sum rate $R$ of the MU-MIMO broadcast system is

$$
R(S N R)=\sum_{k=1}^{K} \log _{2}\left(1+\operatorname{SINR}_{k}\right)
$$

where the signal-to-noise-ratio $S N R=P / \sigma^{2}$. Assume that zero-forcing (ZF) transmit precoding $\mathbf{F}$ is designed based on the quantized channel direction information (CDI). Thus,

$$
\mathbf{F}=\mathbf{H}^{H}\left(\mathbf{H H}^{H}\right)^{-1}, \mathbf{H}=\left[\begin{array}{llll}
\hat{\mathbf{h}}_{1} & \hat{\mathbf{h}}_{2} & \cdots & \hat{\mathbf{h}}_{K}
\end{array}\right]^{T}
$$

where $\tilde{\mathbf{h}}_{k}=\mathbf{h}_{k} /\left\|\mathbf{h}_{k}\right\|_{2}$ is the CDI of the $k$-th user, $\hat{\mathbf{h}}_{k}$ denotes the codeword selected to represent $\tilde{\mathbf{h}}_{k}$, and $\mathbf{H}$ is the quantized CDI matrix. Note that $\mathbf{f}_{k}$ is the normalized $k$-th column of $\mathbf{F}$.

\section{NOVEl ADAPTIVE CODEBOOK TECHNIQUES FOR MU-MIMO-OFDM SYSTEMS}

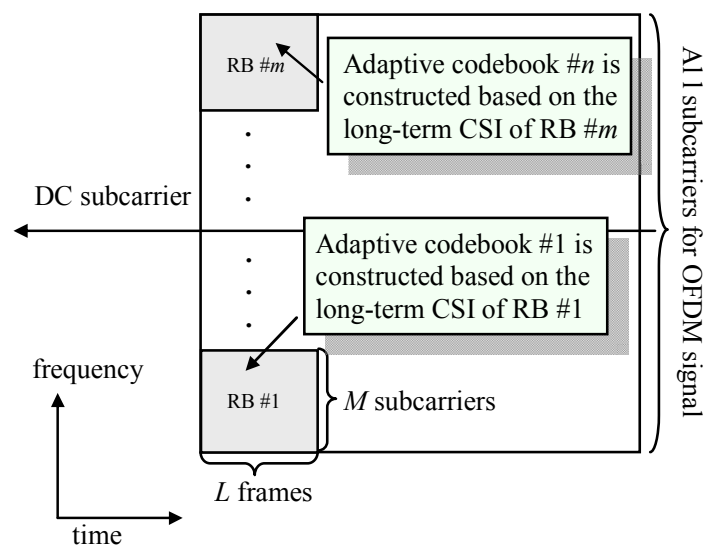

Fig. 1. Adaptive codebook construction in MIMO-OFDM systems

As shown in Fig.1, one two-dimensional resources block (RB) for OFDM signal transmission consists of $L$ frames and $M$ subcarriers where one frame consists of $J$ OFDM symbols. The size of the RB is chosen such that the CSI's in the RB have high time and frequency correlations. Under such a situation, these CSI's can then be characterized using a codebook $\hat{\mathbf{C}}:\left\{\hat{\mathbf{c}}_{1}, \hat{\mathbf{c}}_{2}, \ldots, \hat{\mathbf{c}}_{N}\right\}$ that is constructed to adapt to the long term channel statistics of the RB. The general designing procedure for constructing $\hat{\mathbf{C}}$ is first to start with a root codebook $\mathbf{C}:\left\{\mathbf{c}_{1}, \mathbf{c}_{2}, \ldots, \mathbf{c}_{N}\right\}$ and then transform $\mathbf{C}$ into $\hat{\mathbf{C}}$ according to the chosen channel characteristics.

\section{A. Adaptive Codebook Technique I (using CDI Covariance)}

The long term channel spatial covariance matrix can be employed to construct an adaptive codebook for the CDI in the MU-MIMO-OFDM case.

Similar to the two-step approach for the SU-MIMO single-carrier case in [9], a root codebook is designed for a spatially uncorrelated Rayleigh fading channel in the first step. Then, in the second step, the root codebook is mapped adaptively to a new adaptive codebook such that the new codeword covariance is similar to the CDI covariance.

Take the correlated Grassmannian adaptive codebook design as an example. In the first step, an uncorrelated Grassmannian codebook $\mathbf{C}$ is designed to maximize the minimum chordal distance

$$
\delta_{\min }=\min _{1 \leq i<l \leq N} \sqrt{1-\left|\mathbf{c}_{i}^{H} \mathbf{c}_{l}\right|^{2}}
$$

where $\mathbf{c}_{i}$ is the $i$-th unit-norm codeword in the codebook. The covariance of the root codebook is

$$
\mathbf{R}_{c}=\frac{1}{N} \sum_{i=1}^{N} \mathbf{c}_{i} \mathbf{c}_{i}{ }^{H} \approx \beta \mathbf{I},
$$

where $\mathbf{I}$ is an identical matrix and $\beta$ is a constant.

In the second step, the uncorrelated codebook $\mathbf{C}$ is rotated based on the CDI covariance $\mathbf{R}_{k}$ the $k$-th user to get the adaptive codebook $\hat{\mathbf{C}}_{k}$ for the $k$-th user:

$$
\hat{\mathbf{c}}_{k, q}=\mathbf{R}_{k}^{1 / 2} \mathbf{c}_{q}, \mathbf{R}_{k} \approx \frac{1}{J L M} \sum_{\{l, n \in \in R B} \tilde{\mathbf{h}}_{k}(l, n) \tilde{\mathbf{h}}_{k}^{H}(l, n)
$$

where $\hat{\mathbf{c}}_{k, q}$ is the $q$-th codeword in the adaptive codebook $\hat{\mathbf{C}}_{k}$ and $J L M$ is the total number of channel vectors in one RB. Using (7), the covariance of the adaptive codebook is approximately proportional to the channel spatial correlation:

$$
\mathbf{R}_{k, \hat{c}}=\frac{1}{N} \sum_{q=1}^{N} \hat{\mathbf{c}}_{k, q} \hat{\mathbf{c}}_{k, q}{ }^{H} \approx \beta \mathbf{R}_{k} .
$$

If it is desired, the codeword $\hat{\mathbf{c}}_{k, q}$ can be further normalized as

$$
\tilde{\mathbf{c}}_{k, q}=\frac{\hat{\mathbf{c}}_{k, q}}{\left\|\hat{\mathbf{c}}_{k, q}\right\|_{2}},
$$




\section{B. Adaptive Codebook Technique II (using CDI Mean)}

The long term channel mean can also be employed to construct an adaptive codebook for the CDI. The three-step approach for the SU MIMO single-carrier case in [11] will be extended to deal with the MU MIMO-OFDM case.

Similar to [11], a root codebook with global or local packing is designed in the first step. In the second step, as shown in Fig.2, the center of the root codebook is rotated adaptively to align with the CDI mean $\mathbf{m}_{k}$ in the second step:

$$
\mathbf{m}_{k}=\frac{\widetilde{\mathbf{m}}_{k}}{\left\|\widetilde{\mathbf{m}}_{k}\right\|_{2}}, \quad \widetilde{\mathbf{m}}_{k}=\frac{1}{J L M} \sum_{\{l, n\} \in R B} \tilde{\mathbf{h}}_{k}(l, n)
$$

Codebook rotation can be implemented through multiplying each codeword by a unitary matrix since unitary transform does not change the distance between vectors. Suppose vector $\mathbf{c}_{0}$ is the codebook center before rotation and vector $\tilde{\mathbf{c}}_{0}$ is the target direction for rotation. Then,

$$
\tilde{\mathbf{c}}_{0}=\mathbf{U}_{r o t}\left(\mathbf{c}_{0}, \tilde{\mathbf{c}}_{0}\right) \mathbf{c}_{0}, \mathbf{U}_{r o t}\left(\mathbf{c}_{0}, \tilde{\mathbf{c}}_{0}\right)=\left[\begin{array}{ll}
\tilde{\mathbf{c}}_{0} & \tilde{\mathbf{c}}_{0}^{\perp}
\end{array}\right]\left[\begin{array}{cc}
\mathbf{c}_{0} & \mathbf{c}_{0}^{\perp}
\end{array}\right]^{H}
$$

where $\mathbf{U}_{r o t}$ is the unitary rotation matrix determined by $\mathbf{c}_{0}$ and $\tilde{\mathbf{c}}_{0}$.

In the third step, also shown in Fig. 2, the distance between any codeword and the codebook center is scaled by a common factor $\alpha$ so that the codebook has the optimum coverage and resolution. To implement a codebook scaling, first define a vertex scaling operator $\mathbf{S}_{\text {vertex }}$ which scales a code book whose center is at vertex $\mathbf{e}_{0}=\left[\begin{array}{llll}1 & 0 & \cdots & 0\end{array}\right]$ as

$$
\mathbf{S}_{\text {vertex }}\left(\mathbf{c}_{q} \mid \mathbf{e}_{0}, \alpha\right)=\left[\begin{array}{llll}
\left.\sqrt{1-\alpha^{2}\left(1-\left|c_{q, 1}\right|^{2}\right.}\right) e^{j \theta_{1}} & \alpha c_{q, 2} & \ldots & \alpha c_{q, N_{t}}
\end{array}\right]
$$

where $\alpha$ is the scaling factor and $\mathbf{c}_{q}=\left[\begin{array}{llll}c_{q, 1} & c_{q, 2} & \cdots & c_{q, N_{t}}\end{array}\right]$ is the $q$-th codeword in the codebook and $c_{q, 1}=\left|c_{q, 1}\right| e^{j \theta_{1}}$. It can be easily verified that the codebook center (the reference vertex $\mathbf{e}_{0}$ ) is invariant under the vertex scaling operation. However, the distance between any other codeword to the codebook center is scaled by a factor: $\alpha$. Scaling operation with respect to a general codebook center can be implemented by a composite operation of $\mathbf{U}_{\text {rot }}$ and $\mathbf{s}_{\text {vertex }}$. For example, if we want to scale a codebook whose center is at $\mathbf{c}_{0}$ with a scaling factor $\alpha$. The general scaling operator is defined as

$$
\begin{aligned}
& \mathbf{s}\left(\mathbf{c}_{q} \mid \mathbf{c}_{0}, \alpha\right)=\mathbf{U}_{\text {rot }}\left(\mathbf{e}_{0}, \mathbf{c}_{0}\right) \mathbf{s}_{\text {vertex }}\left(\overline{\mathbf{c}}_{q} \mid \mathbf{e}_{0}, \alpha\right), \\
& -\mathbf{c}_{q}=\mathbf{U}_{r o t}\left(\mathbf{c}_{0}, \mathbf{e}_{0}\right) \mathbf{c}_{q} .
\end{aligned}
$$

In (13), the codebook centered at $\mathbf{c}_{0}$ is rotated to the new center at $\mathbf{e}_{0}$, scaled by the factor $\alpha$, and rotated back to the original center $\mathbf{c}_{0}$.

Combining (11) and (13), the new adaptive codebook is constructed by the following operations:

$$
\hat{\mathbf{c}}_{k, q}=\mathbf{s}\left(\overrightarrow{\mathbf{c}}_{q} \mid \mathbf{m}_{k}, \alpha\right), \quad \overrightarrow{\mathbf{c}}_{q}=\mathbf{U}_{r o t}\left(\mathbf{c}_{0}, \mathbf{m}_{k}\right) \mathbf{c}_{q}
$$

where $\mathbf{U}_{\text {rot }}$ rotates the entire codebook such that the codebook center $\mathbf{c}_{0}$ is aligned with CDI mean $\mathbf{m}_{k}$ and $\mathbf{s}$ scales the entire codebook with respect to the new codebook center $\mathbf{m}_{k}$ with the scaling factor $\alpha$. In this manner, the normalized mean $\mathbf{m}_{c, k}$ of the adaptive codebook in (14) is approximately equal to the CDI mean $\mathbf{m}_{k}$.

$$
\mathbf{m}_{c, k}=\frac{\tilde{\mathbf{m}}_{c, k}}{\left\|\tilde{\mathbf{m}}_{c, k}\right\|_{2}} \simeq \mathbf{m}_{k}, \quad \tilde{\mathbf{m}}_{c, k}=\frac{1}{N} \sum_{k=1}^{N} \hat{\mathbf{c}}_{k}
$$

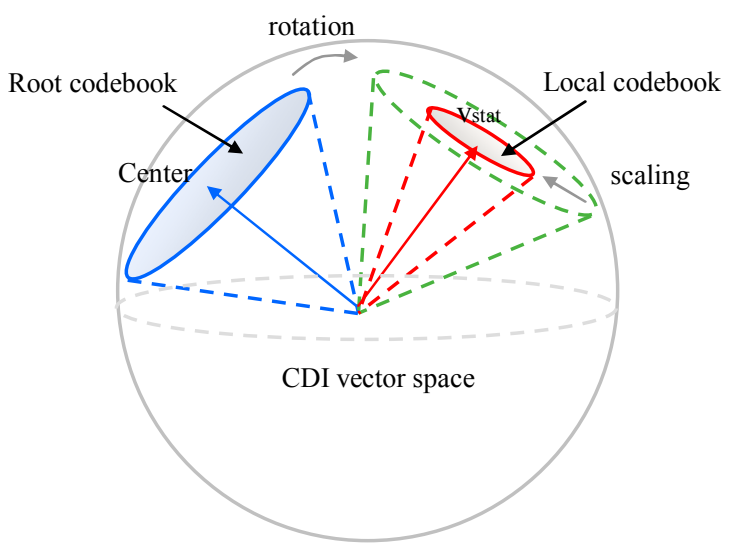

Fig. 2. Adaptive local codebook construction (approach II)

\section{Adaptive Codebook Technique III (using both CDI Covariance and CDI mean)}

In the adaptive codebook technique III, both the first-order statistics (CDI mean) and the second-order statistics (CDI variance) will be exploited to further improve the system performance. The main goal is to have the adaptive codebook satisfies both (8) and (15). Note that the transformation in (8) preserves the CDI covariance but alters the CDI mean. Similarly, the transformation in (15) preserves the CDI mean but alters the CDI covariance. Thus, we cannot just simply combine the two transformations.

To overcome the above mentioned difficulty, we consider the differential channel covariance

$$
\mathbf{R}_{d}=\frac{1}{J L M} \sum_{\{l, n\} \in R B} \mathbf{d}_{k}(l, n) \mathbf{d}_{k}^{H}(l, n)
$$

where $\mathbf{d}_{k}(l, n)=\tilde{\mathbf{h}}_{k}(l, n)-\mathbf{m}_{k}$ (the subscript $k$ is omitted for $\mathbf{R}_{d}$ ). Perform the eigen decomposition of $\mathbf{R}_{d}$

$$
\mathbf{R}_{d}=\mathbf{U}_{d} \boldsymbol{\Lambda}_{d} \mathbf{U}_{d}{ }^{H}
$$

where $\mathbf{U}_{d}$ is the eigenvector matrix of $\mathbf{R}_{d}$ and $\boldsymbol{\Lambda}_{d}$ is a diagonal matrix where its diagonal elements are the eigenvalues of $\mathbf{R}_{d}$. Also compute the codeword covariance $\mathbf{R}_{c}$ of the root codebook $\mathbf{C}$

$$
\mathbf{R}_{c}=\frac{1}{N} \sum_{k=1}^{N} \mathbf{c}_{k} \mathbf{c}_{k}{ }^{H}
$$

and perform the eigen decomposition of $\mathbf{R}$ 


$$
\mathbf{R}_{c}=\mathbf{U}_{c} \boldsymbol{\Lambda}_{c} \mathbf{U}_{c}^{H}
$$

where $\mathbf{U}_{c}$ is the eigenvector matrix of $\mathbf{R}_{c}$ and $\boldsymbol{\Lambda}_{c}$ is a diagonal matrix where its diagonal elements are the eigenvalues of $\mathbf{R}_{c}$. Then, the adaptive codebook is

$$
\hat{\mathbf{c}}_{k, q}=\tilde{\mathbf{c}}_{k, q} /\left\|\tilde{\mathbf{c}}_{k, q}\right\|_{2}, \quad \tilde{\mathbf{c}}_{k, q}=\mathbf{c}_{k, \text { iiff }}+\mathbf{m}_{k}
$$

where

$$
\mathbf{c}_{k, d i f f}=\mathbf{U}_{d} \boldsymbol{\Lambda}_{d}{ }^{1 / 2} \boldsymbol{\Lambda}_{c}^{-1 / 2} \mathbf{U}_{c}{ }^{H} \mathbf{c}_{k} .
$$

Note that, using (20) and (21), the differential channel covariance is preserved:

$$
\frac{1}{N} \sum_{k=1}^{N} \mathbf{c}_{k, d i f f} \mathbf{c}_{k, d i f f}^{H} \approx \mathbf{R}_{d},
$$

and the channel mean is also preserved:

$$
\frac{1}{N} \sum_{k=1}^{N} \tilde{\mathbf{c}}_{k, q} \approx \mathbf{m}_{k} .
$$

\section{Simulation Results}

The sum rate performances of the proposed adaptive codebook techniques for MU-MIMO-OFDM broadcast systems with the LTE profile are investigated The base station equipped with four transmit antennas will transmit to users with one receive antenna per user. The spatial MIMO channel model used in simulation is the suburban macrocell in [14] without considering the long distance path loss. Each LTE downlink frame is $10 \mathrm{~ms}$ long and consists of 10 subframes. Define the time duration for each subframe (1 ms) as the Transmission Time Interval (TTI). Each TTI comprises 14 OFDM symbols $(J=14)$. The channel coherent time in simulation is around 20 TTI's. The FFT size of the OFDM system is 1024. The subcarrier spacing is $15 \mathrm{KHz}$. The number of data subcarriers carried on each preamble is 600 .

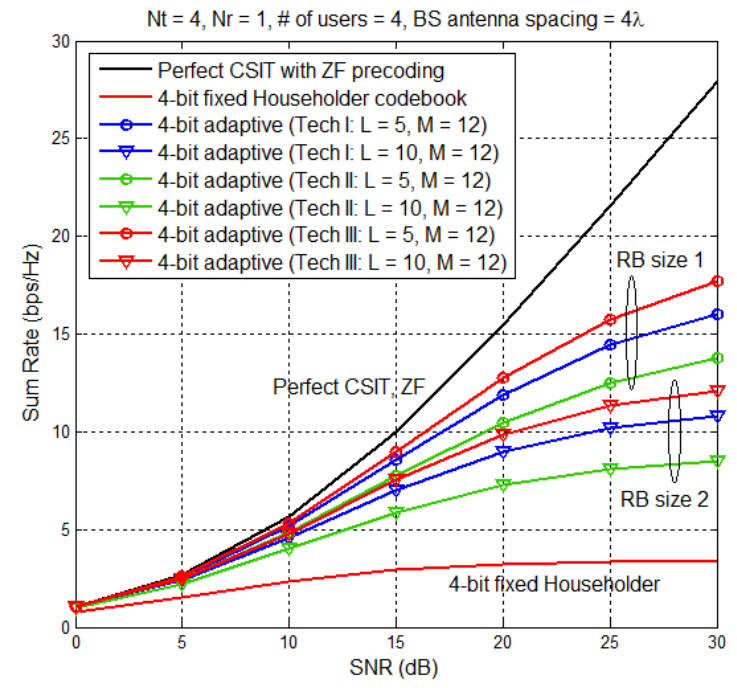

Fig. 3. Sum rate performance of the three adaptive codebook techniques $\mathrm{Nt}=4, \mathrm{Nr}=1$, Number of users $=4, \mathrm{BS}$ antenna spacing $=4 \lambda$

The sum rate performances of the three adaptive codebook approaches for different $\mathrm{RB}$ sizes are investigated and compared with the fixed codebook result and the perfect CSIT result. The fixed codebooks used for different users should be different and are generated by multiplying the 4-bit Householder codebook [3] by different unitary matrices. The root code book used for the adaptive codebook construction is a 4-bit Grassmannian codebook [15]. The adaptive codebooks for each RB will be constructed based on the long term CSI's (CDI mean and/or covariance) which are assumed to be perfectly known at both receiver and transmitter. The codebook scaling factor used in the adaptive codebook technique II is $\alpha=0.2$. Two different sizes of RBs are considered in simulation. One is 5 TTI's $\times 12$ subcarries $(L=$ $5, M=12)$ and the other is 10 TTI's $\times 12$ subcarries $(L=10$ and $M=12$ ).

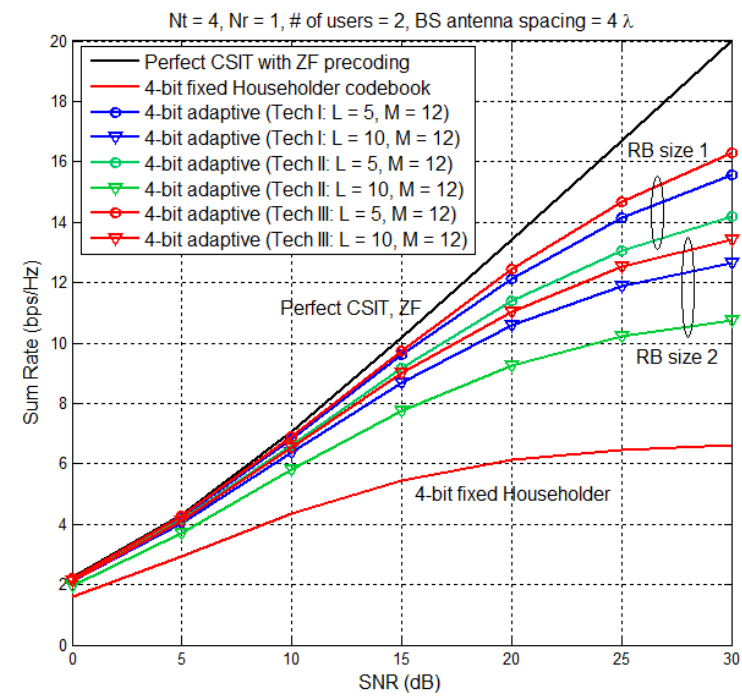

Fig. 4. Sum rate performance of the three adaptive codebook techniques

$\mathrm{Nt}=4, \mathrm{Nr}=1$, Number of users $=2, \mathrm{BS}$ antenna spacing $=4 \lambda$

In Fig.3, the antenna spacing at the base station is $4 \lambda$ ( $\lambda$ is the wavelength of the carrier) and four users are randomly generated in a cell. In Fig. 4, the antenna spacing remains as $4 \lambda$ but only two users are considered. In Fig. 5, the antenna spacing is reduced to $1.5 \lambda$ and only two users are considered. From the simulation results shown in Figs.3-5, the sum rate performances of the three adaptive codebook techniques are much better than the fixed codebook solution. In addition, technique III outperforms techniques I and II (with a significant gain compared to technique II and a medium gain compared to technique I) for all simulations. Comparing the results of two TTI's, we conclude that reducing the RB size (increase the instant CSI correlation in the RB) will improve the sum rate performance but add more feedback overhead.

Remarkable observations can also be made by comparing Fig. 3 to Fig.4. For perfect CSI, the 4-user result is better than the 2-user result since the transmitter has 4 antennas and the system can support up to 4 users. However, although the sum rate increases as the number of users increases (up to 4), the 4-user sum rate cannot reach to twice of the 2-user sum rate because there is diversity gain in the 2-user case but not in the 4-user case. 
For fixed codebook, unlike perfect CSI, the 4-user sum rate is worse than the 2-user sum rate because fixed codebook provides a poor CDI resolution and the resulting zero forcing precoder cannot cancel the MU interferences well. Thus, more users generate more MU interferences. The MU interferences in the 4-user case are so strong that the advantages of multiplexing totally disappear. Instead, the sum rate decreases as the number of users increases.

Similar to fixed codebook, the 4-user sum rate is worse than the 2-user sum rate in the adaptive technique II simulation. For the adaptive technique I simulation, the 2-user sum rate is more or less equal to the 4-user sum rate when $\mathrm{L}=5$. However, for the adaptive III simulation, the 4-user sum rate is larger than the 2-user when $\mathrm{L}=5$ which is similar to the perfect CSI simulation. Thus, we can conclude again that, among the three techniques, III is the best and II is the worst.

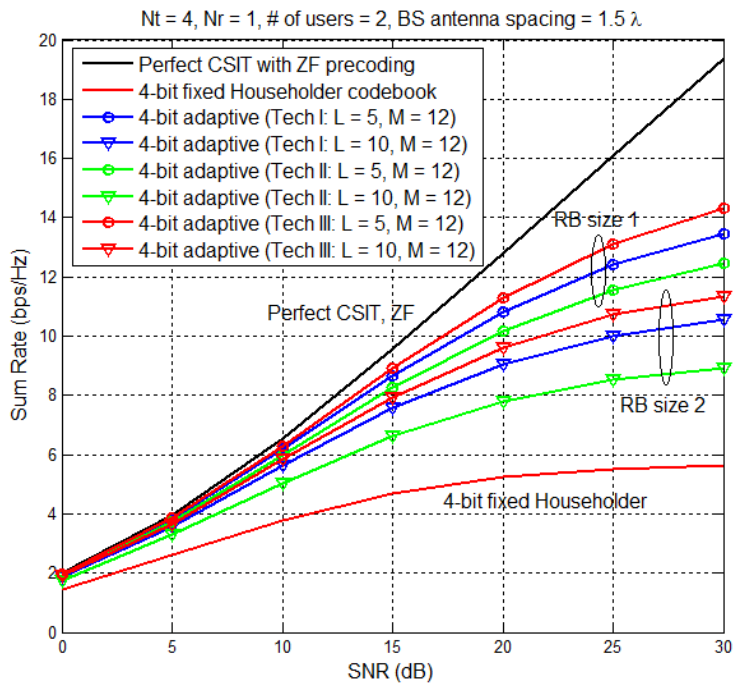

Fig. 5. Sum rate performance of the three adaptive codebook techniques

$\mathrm{Nt}=4, \mathrm{Nr}=1$, Number of users $=2, \mathrm{BS}$ antenna spacing $=1.5 \lambda$

Comparing Fig. 4 to Fig.5, the $4 \lambda$ result is better than the $1.5 \lambda$ result for perfect CSI. This is due to the fact that the channels in the $4 \lambda$ case are less correlated than those in the $1.5 \lambda$ case. For the same reason and like perfect CSI, the $4 \lambda$ result is better than the $1.5 \lambda$ result for all limited feedback simulation (including fixed codebook and three adaptive codebook techniques). What we are interested here is the performance improvement of adaptive codebooks over the fixed codebook. For a single user scenario (not shown here), we observed the improvement in the high correlation environment $(1.5 \lambda)$ is larger than that in the low correlation

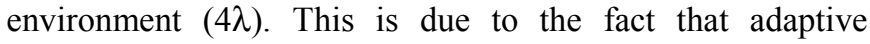
techniques are generally more effective in high correlation environments. However, we don't find the same observation here in the multiuser scenario because the multiuser interference generated by imperfect zero forcing eliminates the merits brought up by strong correlation for adaptive codebooks.

\section{CONCLUSION}

In this paper, we investigate the adaptive codebook-based limited feedback techniques for MU-MIMO-OFDM broadcast systems. Using channel covariance and mean respectively, techniques I and II are extensions of two adaptive codebook techniques proposed for SU-MIMO single-carrier systems. Technique III is a new proposal wherein both channel covariance and mean are exploited in the adaptive codebook construction to further improve the performance. The sum rate performances of the three adaptive codebook techniques in MU-MIMO OFDM systems are investigated through simulation based on the LTE system profile. The adaptive codebook approaches have significant sum rate gain compared to the fixed codebook solution. The novel proposed technique III outperforms substantially technique I and II in sum rate performance. We can conclude that adaptive codebook techniques are important for FDD MU-MIMO-OFDM systems and techniques using both the first order and the second order channel statistics outperform techniques just using either the first order or the second order channel statistics.

\section{REFERENCES}

[1] Wi-Fi Alliance. Wi-Fi certified 802.11n draft 2.0: longer-range, faster-throughput, multimedia-grade Wi-Fi networks, 2007.

[2] IEEE $802.16 \mathrm{~m}$ system description document (SDD), Sept. 2009.

[3] 3GPP TS 36.211 v8.0.0. $3^{\text {rd }}$ Generation Partnership Project Technical Specification Group Radio Access Network Evolved Universal Terrestrial Radio Access (E-UTRA) Physical channels and modulation (Release 8), Sept. 2007.

[4] David J. Love, Robert W. Heath, Jr et al. "An overview of limited feedback in wireless communication systems," IEEE Journal On Selected Areas In Communications, vol. 26, no.8, pp. 1341-1364, Oct. 2008.

[5] David J. Love, Robert W. Heath, Jr et al., "Grassmannian beamforming for multiple-input multiple-output wireless systems," IEEE Trans. On Information Theory, vol. 49, no.10, pp. 2753-2747, Oct. 2003.

[6] J. C. Roh and B. D. Rao, "Transmit beamforming in multiple-antenna systems with finite rate feedback: a VQ-based approach," IEEE Trans. On Information Theory, vol. 52, no. 3, pp. 1101-1112, Mar. 2006.

[7] D. J. Love and R. W. Heath, Jr., "Equal gain transmission in multiple-input multiple-output wireless systems," IEEE Trans. On Communications, vol. 51, no. 7, pp. 1102-1110, Jul. 2003.

[8] T. Strohmer and R. W. Heath Jr., "Grassmannian frames with applications to coding and communications," Applied and Computational Harmonic Analysis, vol. 14, no. 3, pp. 257-275, May. 2003.

[9] David J. Love and Robert W. Heath, Jr., "Limited feedback diversity techniques for correlated channels," IEEE Trans. On Vehicular Technology, vol. 55, no. 2, pp. 718-722, Mar. 2006.

[10] Roopsha Samanta and Robert W. Heath, Jr, "codebook adaptation for quantized MIMO beamforming systems," Proc. IEEE Asilomar Conf. Signals, Systems, and Computers, pp. 376-380, Nov. 2005.

[11] Vasanthan Raghavan, Robert W. Heath, Jr. et al, "Systematic codebook designs for quantized beamforming in correlated MIMO channels," IEEE Journal On Selected Areas In Communications, Vol. 25, no. 7, pp. 1298-1310, Sept. 2007.

[12] Nihar Jindal, "MIMO broadcast channel with finite-rate feedback," IEEE Journal On Information Theory, Vol. 52, no. 11, pp. 5045-5060, Nov. 2006.

[13] Allen Gersho and Robert M. Gray. Vector quantization and signal compression, Kluwer Academic Publishers, 1992.

[14] Project 802.16m Evaluation Methodology document. 2008.

[15] http://cobweb.ecn.purdue.edu/ djlove/grass.html 